\title{
The effectiveness of the biannual application of silver nitrate solution followed by sodium fluoride varnish in arresting early childhood caries in preschool children: study protocol for a randomised controlled trial
}

\author{
Chun-Hung Chu*, Sherry Shiqian Gao, Samantha KY Li, May CM Wong and Edward CM Lo
}

\begin{abstract}
Background: The application of $38 \%$ silver diamine fluoride (SDF) has been shown to be effective in arresting early childhood caries (ECC). Since SDF is not available in certain countries, some dentists use adjunctive application of $25 \%$ silver nitrate $\left(\mathrm{AgNO}_{3}\right)$ and $5 \%$ sodium fluoride $(\mathrm{NaF})$ to arrest ECC. This randomised controlled trial will systematically compare the efficacy of a $25 \% \mathrm{AgNO}_{3}$ solution followed by $5 \% \mathrm{NaF}$ varnish with that of a $38 \% \mathrm{SDF}$ solution in arresting ECC when applied at half-yearly intervals over a 30-month period.

Methods/Design: This study is a randomised, double-blinded, non-inferiority clinical trial. The hypothesis tested is that adjunctive application of $25 \% \mathrm{AgNO}_{3}$ followed by $5 \% \mathrm{NaF}$ is at least not appreciably worse than a $38 \% \mathrm{SDF}$ in arresting ECC. Approximately 3100 kindergarten children aged 3-4 years will be screened and at least 1070 children with caries will be recruited. This sample size is sufficient for an appropriate statistical analysis (power at $90 \%(\beta=0.10)$ with a 2 -sided type-I error of $a=0.05)$, allowing for an overall $20 \%$ drop-out rate. The children will be randomly allocated into 2 groups to treat their caries over a 30-month period:

Group A - biannual adjunctive application of a $25 \% \mathrm{AgNO}_{3}$ solution and a $5 \% \mathrm{NaF}$ varnish, and Group B - biannual adjunctive application of a 38 \% SDF solution followed by a placebo varnish.

Clinical examinations will be conducted at 6-month intervals. Primary outcome measured is the number of active caries surfaces which are arrested. Information on confounding factors such as oral hygiene habits will be collected through a parental questionnaire.

Discussion: We expect that adjunctive application of $25 \% \mathrm{AgNO}_{3}$ solution and $5 \% \mathrm{NaF}$ varnish and of $38 \% \mathrm{SDF}$ solution can both effectively arrest ECC. Lower concentrations of silver and fluoride are contained in $25 \% \mathrm{AgNO}_{3}$ and $5 \% \mathrm{NaF}$, respectively, than in $38 \% \mathrm{SDF}$; therefore, $\mathrm{AgNO}_{3} / \mathrm{NaF}$ are more favourable for use in young children. Because its use for caries management is painless, simple, low-cost, and approved in many countries, $\mathrm{AgNO}_{3} / \mathrm{NaF}$ could be widely recommended and promoted as an alternative treatment to conventional invasive management of ECC.
\end{abstract}

Trial registration: ClinicalTrials.gov: NCT02019160. Date of registration: 11 December 2013.

Keywords: Silver nitrate, Fluoride varnish, Silver diamine fluoride, Caries

\footnotetext{
* Correspondence: chchu@hku.hk

Faculty of Dentistry, University of Hong Kong, 34 Hospital Road, Hong Kong, SAR, China
} 


\section{Background}

\section{Early childhood caries}

Early childhood caries (ECC) is defined as the presence of one or more decayed, missing (due to dental caries), or filled tooth surfaces in the primary teeth of a child of 71 months of age or under [1]. Despite advances in clinical care and dental research, ECC remains a challenging problem. The United States (US) Centers for Disease Control and Prevention has reported that $28 \%$ of all US toddlers and preschoolers are affected by ECC and that nearly half of US children have ECC before entering kindergarten [2]. ECC cause pain and infection, and advanced caries will progress into the tooth pulp to eventually form a dental abscess [3]. If cases remain untreated they lead to tooth loss, which affects dentition. Poor dentition significantly affects children's nutrition and consequently their growth, development, and general health. Socially disadvantaged children, such as those from poor families and those whose parents have low educational levels, are disproportionately affected [4, 5]. The FDI World Dental Federation Oral Health Atlas reported that millions of children are suffering from untreated ECC; however, with its great shortage of community dentists, the present dental care delivery system cannot cope with this high ECC prevalence [6]. Moreover, prevailing restorative methods for preventing and treating ECC are neither available nor affordable to children from disadvantaged families, who exhibit a particularly high caries risk [7]. Although there have been efforts to treat ECC in developing areas (e.g., using mobile dental clinics with portable equipment), in most cases the technology has proven to be too complicated for sustained use [8]. The cost of basic sets of instruments, dental materials and infection-control products is also too high. In this situation, a new approach for managing ECC is needed. This study uses a non-operative strategy for caries management in preschool children.

\section{Silver diamine fluoride}

Fluoride was introduced to prevent caries more than 50 years ago [9]. Clinical studies have demonstrated that using $38 \%$ silver diamine fluoride (SDF) can prevent and arrest ECC [10-15]. A literature review of SDF suggested that $38 \%$ SDF can be an effective agent in preventing new caries and arresting dental caries in the primary teeth of children [16]. Another review concluded that SDF is an effective, efficient, and equitable caries-control agent [17]. Milgrom and Chi [18] advocated SDF therapy as an important prevention-centred caries management strategy during critical early childhood periods. The clinical trial by dos Santos et al. [19] concluded that $38 \%$ SDF was better than interim

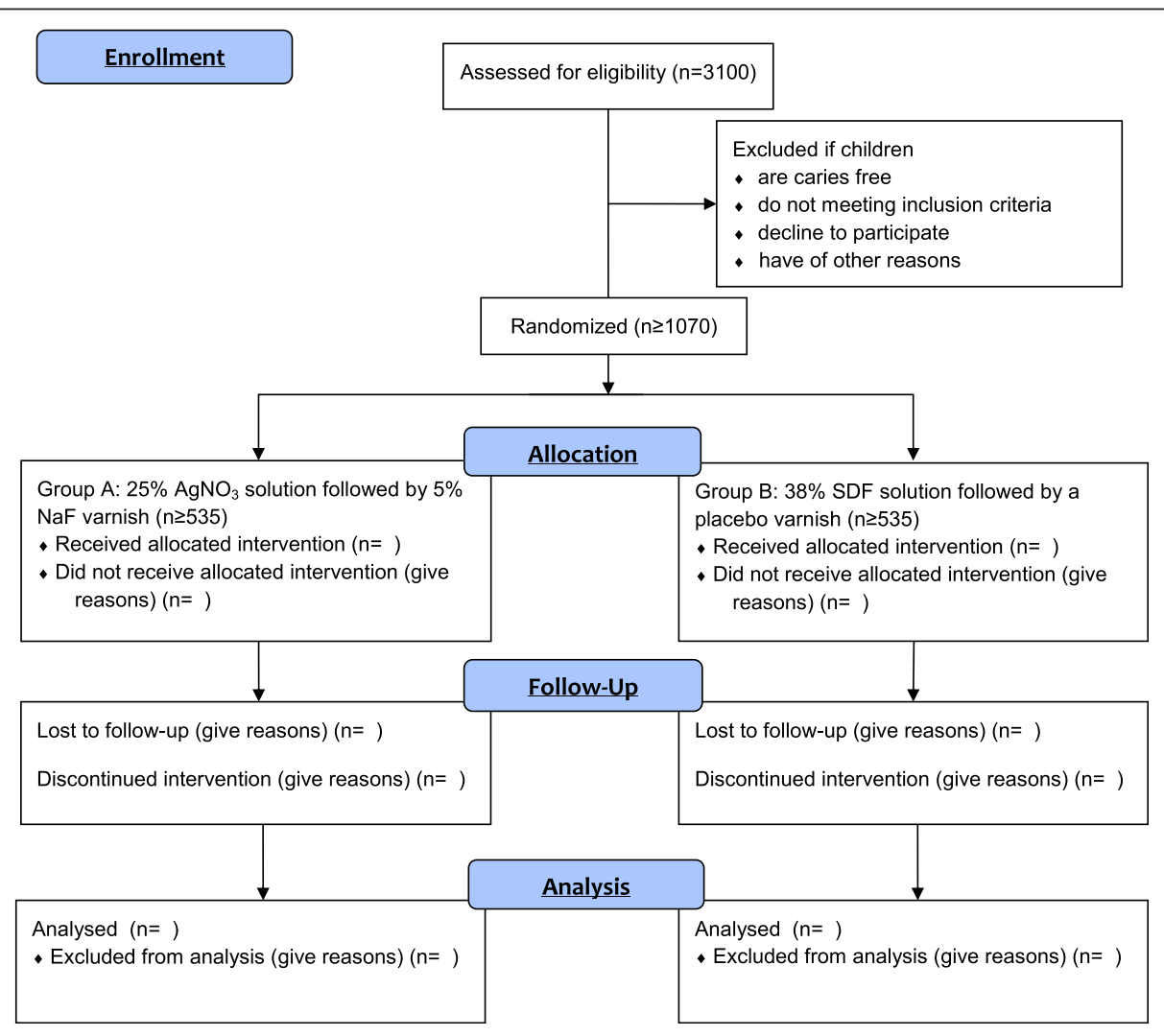

Fig. 1 Flow diagram of the clinical trial 
restorative treatment with a glass ionomer for arresting ECC. Nevertheless, 38 \% SDF exhibits a high fluoride concentration $(44,800 \mathrm{ppm})$, which has concerned some researchers because of the possible onset of dental fluorosis [20]. Although SDF is used in countries in Asia and South America, SDF has not yet been approved for use in many European countries or in the USA [21].

\section{Adjunctive application of silver nitrate solution and sodium fluoride varnish}

Although dental fluorosis can develop with increased fluoride dosage, fluoride use under professional instruction is generally safe and effective even in young children [9]. Sodium fluoride $(\mathrm{NaF})$ varnish at $5 \%$ contains $22,600 \mathrm{ppm}$ of fluoride ions and is a common professionally applied topical agent in caries management. A literature review on the use of $\mathrm{NaF}$ varnish in managing ECC in children found that fluoride varnish appears to be the easier caries prevention method for both the dentist and the child [22]. However, NaF varnish is not effective in arresting dentine caries [10, 13]. Duffin [23] proposed using silver and fluoride ions from $25 \%$ silver nitrate $\left(\mathrm{AgNO}_{3}\right)$ solution followed by $5 \% \mathrm{NaF}$ varnish to arrest caries. A solution of $25 \% \mathrm{AgNO}_{3}$ and $5 \% \mathrm{NaF}$ varnish are accepted by most countries and their corresponding authorities such as the US Food and Drug Administration [23]. One laboratory study has suggested that $25 \% \mathrm{AgNO}_{3}$ solution and $5 \% \mathrm{NaF}$ varnish may be as effective as $38 \%$ SDF in arresting dentine caries [24, 25]. However, a discussion of the use of $\mathrm{AgNO}_{3}$ and $\mathrm{NaF}$ by the Oregon Dental Association concluded that more research demonstrating efficacy and safety is required to meet the standard for evidence-based dentistry [21]. A literature search on PubMed on 1 December 2014, however, found that no research group has thus far published a welldesigned study on the effectiveness of $\mathrm{AgNO}_{3}$ and $\mathrm{NaF}$ in the control of ECC.

\section{Objective}

The overall objective of the randomised controlled trial is to systematically compare the effectiveness of a $25 \%$ $\mathrm{AgNO}_{3}$ solution followed by $5 \% \mathrm{NaF}$ varnish with that of a $38 \%$ SDF solution in arresting caries teeth among preschool children when applied at half-yearly intervals over a 30 -month period.

\section{Hypothesis}

In this non-inferiority randomised controlled trial, the hypothesis tested is that the biannual topical application of $25 \% \mathrm{AgNO}_{3}$ solution followed by $5 \% \mathrm{NaF}$ varnish is at least not appreciably worse than a $38 \%$ SDF solution in terms of caries arrest in the primary teeth of preschool children.

\section{Methods/Design \\ Trial design}

This is a randomised, double-blind, non-inferiority clinical trial. The extension of the Consolidated Standards of Reporting Trials 2010 Statement will be followed [26]. The flowchart of this study is shown in Fig. 1.

\section{Setting}

Kindergartens in Hong Kong who have joined our outreach dental service will be invited to join this study. Preschool children aged 3-4 years who have tooth decay and are attending the first year of kindergarten will be invited to join this study. An invitation letter will be sent to the parents explaining the purpose and procedures of this study. Written parental consent will be obtained before they are included in this clinical trial. We have trained an outreach team comprised of a dentist and a dental assistant to assess caries prevention and the caries-arresting effects of different treatments in preschool children in the kindergartens.

\section{Participants}

The children included in the study should: a) be aged 34 years and are attending the first year of kindergarten, b) be generally healthy, c) have parental consent, and d) have at least 1 tooth with untreated caries with cavitation that extends into the dentine at baseline examination. Children who are uncooperative and difficult to manage, with severe forms of hypoplasia of fluorosis or other oral diseases, wearing orthodontic devices or under dental treatment, have major systemic diseases, or are on long-term medication will be excluded.

\section{Recruitment and screening}

Oral health education will be provided to the children in the participating kindergartens, followed by a baseline oral examination. Potential participants will be recruited by trained field workers of the outreach team after the initial oral examination.

\section{Clinical examination}

A clinical examination of the participating children will be performed in the kindergarten, primarily through a careful visual inspection with the aid of a disposable World Health Organisation Community Periodontal Index (CPI) probe (405/WHO probe, Otto Leibinger, Mühlheim, Germany) and a front-surface dental mirror with light-emitting diode intra-oral illumination (MirrorLite, Kudos Crown Limited, Hong Kong, China). The oral hygiene status will be measured using the visible plaque index (VPI). The buccal and lingual surfaces of 6 index teeth $(55,51,63,71,75$, and 83$)$ will be examined. The presence or absence of visible plaque on the caries surface will also be recorded. 
The tooth status (decayed, missing, filled surfaces (dmfs) score), tooth discolouration, and hyper-mobility will be recorded. Teeth with a caries lesion extending into the pulp or signs suggesting that the teeth are nonvital, such as tooth discolouration, hyper-mobility or abscess, will not be included in this study.

The caries will be diagnosed at the cavitation level. The carious lesion will be gently explored with the CPI probe in the centre of the lesion. Great care will be taken to avoid tooth damage during the probing. A lesion will be recorded as active if softness is detected upon gentle probing. If the dentine surface is hard to probing, it will be classified as an arrested caries [11-13, 15, 27]. All surfaces (i.e., buccal, lingual, mesial, distal, and occlusal for posterior teeth) of each tooth will be assessed. Active caries at the baseline that become arrested in the follow-up examinations will be used as the main treatment outcome.

A designated experienced outreach dentist will be the examiner throughout the 30-month study. The intraexaminer agreement on the plaque and caries assessment will be monitored and conducted in $10 \%$ of the children at each stage of the study.

\section{Questionnaire survey}

A validated parental questionnaire [4,5] regarding their children's oral hygiene habits (e.g., tooth-brushing and flossing), parent-assisted tooth-brushing, fluoride agent use (e.g., fluoride toothpaste and fluoride mouthwash), when brushing with fluoride toothpaste commenced, fluoride concentration in the used toothpaste, dental visit behaviour, snacking habits, parental educational level, family total income, and family status (single-parent or 2-parent households) will be administered at baseline and again at the 18-month and 30-month follow-up visits (Additional file 1). The questionnaires will also assess parental satisfaction with their child's oral health and dental aesthetics. In the follow-up questionnaires, questions will also be asked about the potential post-treatment complications of the SDF application, such as pain in the treated tooth and gingival irritation around the treated tooth.

\section{Randomisation and treatment allocation Allocation concealment}

The participating children with caries will first be categorised as either: (1) having a high caries rate (or severe early childhood caries, as defined by the American Academy of Paediatric Dentistry [1]), which is defined as the presence of more than 3 untreated caries surfaces, or (2) having a low caries rate. The children will then be allocated by a stratified randomisation method using a computer-generated random-number table into the following 2 groups:
Group A - biannual topical application of $25 \% \mathrm{AgNO}_{3}$ solution followed by $5 \% \mathrm{NaF}$ varnish; and Group B - biannual topical application of 38 \% SDF solution followed by a placebo varnish.

\section{Blinding}

In this double-blind randomised clinical trial, the group allocation will be conducted by a dental assistant while the examiner will not know the allocation information. The examiner will not be informed of the treatment group allocation of the children and the children will not know the solution or varnish they receive throughout the study. The solution and varnish will be applied after the oral examination by another operator.

\section{Interventions}

An independent operator will use a micro-brush to apply the appropriate solution and varnish according to the assigned treatment group after the oral examination. The Group A children will receive a $25 \% \mathrm{AgNO}_{3}$ solution (25\% silver nitrate, Gordon Labs, Carson, CA, USA) followed by a $5 \% \mathrm{NaF}$ varnish (Duraphat Varnish, Colgate-Palmolive, Endicott, NY, USA). The Group B children will receive a $38 \%$ SDF solution (Saforide, Toyo Seiyaku Kasei Co., Osaka, Japan) followed by a placebo petroleum jelly varnish (Vaseline, Unilever, Englewood Cliffs, NJ, USA). The children in both groups will be instructed not to eat or drink for an hour after the application.

There is no adverse reaction of these two treatments, except the caries surfaces will become hard and black if the treatment has successfully arrested the caries lesions.

After the baseline examination, a report on the child's oral health status will be provided to the parents with a note asking them to report to the study dentist if there are any side/adverse effects on the treated tooth and surrounding gum after treatment. During the follow-up treatments, the dentist will also look for related side/adverse effects, including blackening of the arrested caries surface, discoloured tooth, tooth hyper-mobility, and abscess formation.

\section{Follow-up evaluation}

The follow-up oral examinations will be conducted every 6 months in the kindergarteners for 30 months by the same examiner using the same equipment, procedure, and diagnostic criteria as those used in the baseline examination. The oral hygiene and caries status of the child will be recorded. In addition, the presence of visible plaque and status of the included caries surfaces will be assessed. The examiner will not be the operator for the fluoride application. 


\section{Outcome measure}

The outcome measure (primary endpoint) is the number of soft (active) caries surfaces that become arrested (hardened) after 30 months. The outcome measure will be administered at baseline and at the 6-monthly followup examinations. Children and their parents have the right to receive dental treatment by other dentists. To our experience from previous clinical trials [11, 12], almost all participants will not be cared by other dental personnel, and we will include questions about the receipt of other dental treatment in baseline and follow-up interviews.

\section{Sample size and power calculation}

This non-inferiority trial aims to measure the anti-caries efficacy of a $25 \% \mathrm{AgNO}_{3}$ solution followed by $5 \% \mathrm{NaF}$ varnish versus a $38 \%$ SDF solution with a noninferiority margin of -0.5 for the difference in mean number of arrested caries surfaces, which is considered clinically negligible (corresponding to an effect size = 0.25 , assuming a standard deviation of 2.5 and true difference of 0) [11]. The estimated sample size, calculated with the help of a statistician, is based on the lower limit of the 2 -sided $95 \%$ confidence interval for the difference being set above the non-inferiority margin $(-0.5)$, and statistical power of the study being set at $90 \%(\beta=0.10)$. The total required number of preschool children with caries will be 856 calculated with the use of statistical power analyses software, G*Power (version 3.1.7; Franz Faul, Universität Kiel, Kiel, Germany). Anticipating the caries prevalence to be $35 \%$ [28] and the overall 30month drop-out rate to be $20 \%$ [11], the initial sample size for screening should thus be 3058 with a total of 1070 kindergarten children being recruited in the 2 study groups at the baseline examination.

\section{Statistical methods}

A data management and statistical analysis protocol has been developed for the clinical trial. This details the procedures for data entry, management and cleaning, and data analysis.

The collected data will be entered into an Excel file (Microsoft Corp., Redmond, WA, USA) by two persons, and the data will be compared to minimise data entry errors. The intra-examiner agreement in caries diagnoses will be assessed by Cohen's Kappa statistics. The level of statistical significance for all 2-sided tests will be set at 0.05 . For the 1 -sided tests, the level of statistical significance will be set at 0.025 .

An intention-to-treat analysis will be performed in this study. We expect that the drop-out rates of the two groups will be similar because the treatment protocols and treatment outcomes (arrested caries with blackening of the lesion) are the same. If the number of children who strayed from the protocol (for instance, by not adhering to the prescribed 6-month intervention, or by being withdrawn from active treatment) are different between the 2 groups, a per-protocol analysis will be performed. In that case, only the patients who complete the entire clinical trial according to the protocol will be counted towards the final results.

\section{Subject-level analysis}

Although the data for the primary outcome itself may not be normally distributed [11], making use of the central limit theorem with the relatively large sample size that will be recruited in this research, the distribution of the mean would be normal. Non-inferiority of the $25 \%$ $\mathrm{AgNO}_{3}$ solution followed by $5 \% \mathrm{NaF}$ varnish to $38 \%$ SDF solution can be claimed if the lower limit of the confidence interval for the difference in the mean number of arrested surfaces is greater than the noninferiority margin of -0.5 . This test for non-inferiority will only be performed for the primary endpoint, the number of arrested surfaces after 30 months. Furthermore, a Student's $T$ test will be performed to study the effects of the treatment on the number of arrested caries surfaces at the 18-month examination. This research will also study the effect modification. The variables that are considered to possibly modify the treatment effects on the outcome variable include the patient's gender, baseline caries experiences, treatment group assignment, oral hygiene habits, fluoride agent use, parent-assisted tooth-brushing, dental visit behaviour, snacking habits, parental educational level, total family income, and family status (single-parent or two-parent households).

Besides the primary outcome, Student's $T$ tests will be also used to test the between-group differences regarding the number of new caries surfaces, increment in the number of non-vital teeth, and number of hyper-mobile teeth across the follow-up examinations.

\section{Surface-level analysis}

To compare the differences in the time to caries arrest between the two treatment groups at the tooth surface level, a survival analysis will be adopted for the intervalcensored data (because the time to arrest cannot be observed exactly but falls in the interval between the two examinations); this analysis will account for the possible correlation (clustering) between the observations of multiple surfaces from the same child. The statistical software SAS 9.2 for Windows (SAS Institute Inc., Cary, $\mathrm{NC}$, USA) will be used for the analyses above.

\section{Ethical considerations}

Ethics approval was obtained from the Institutional Review Board of the University of Hong Kong/Hospital Authority Hong Kong West Cluster (HKU/HAHKW 
IRB) (IRB reference number: UW 13-569). Informed consent will be sought from parents of each participating child prior to participation in the trial. In general, the trial will pose minimal risks to the participating children. In order to minimise risk, careful training has been provided to the field workers. A monitoring system is in place for potential adverse and serious adverse events, with a protocol for the management of these events and reporting at appropriate times to the HKU/HAHKW IRB.

All data from the baseline and follow-up examinations will be reviewed on a continuous basis by unblinded members (CHC and ECML) of the team to check on quality. The trial statisticians (SKYL and MCMW) will be asked to review the data at key points during the life of the trial. Reports will be submitted to the HKU/HAHKW IRB every 6 months for external quality control. All serious adverse events will be reported to the HKU/HAHKW IRB within 48 hours of being reported to the trial team.

\section{Discussion}

This study is a non-inferiority clinical trial aiming to investigate whether biannual topical application of $25 \%$ $\mathrm{AgNO}_{3}$ solution followed by $5 \% \mathrm{NaF}$ varnish is at least not appreciably worse than 38 \% SDF solution in arresting caries in the primary teeth of children. The estimated sample size is based, with the help of a statistician, on the lower limit of the 2-sided $95 \%$ confidence interval for the difference being set above the non-inferiority margin $(-0.5)$ and statistical power of the study being set at $90 \%(\beta=0.10)$. As no other similar non-inferiority clinical trial has been conducted in this area, the non-inferiority margin of this trial cannot be inferred from previous studies. Since the number of dental caries surfaces is the integer and the hypothesis of this study is that $25 \% \mathrm{AgNO}_{3}$ solution followed by $5 \% \mathrm{NaF}$ varnish is at least not appreciably worse than $38 \%$ SDF solution in arresting caries, the difference between the results (the number of caries arrested surfaces) is estimated to be between -1 to 0 (Group A minus Group B). After discussing with several experienced statisticians, we finally determined the non-inferiority margin of this study to be -0.5 , which is the middle value between -1 and 0 .

The evaluation will be conducted at 18-month and 30month follow-up. Non-inferiority testing will only be performed for the primary endpoint, the number of arrested surfaces after 30 months. Additionally, a Student's $T$ test will be performed to study the effects of the treatment on the number of arrested caries surfaces at the 18-month examination. As different variables might provide different levels of influence on outcome variables, this research will also study the effect modification. The variables that are considered to possibly modify the treatment effects on the outcome variable include the patient's gender, baseline caries experiences, treatment group assignment, oral hygiene habits, fluoride agent use, parent-assisted tooth-brushing, dental visit behaviour, snacking habits, parental educational level, total family income, and family status (single-parent or two-parent households).

We expect that topical application of $25 \% \mathrm{AgNO}_{3}$ solution followed by $5 \% \mathrm{NaF}$ varnish and of $38 \% \mathrm{SDF}$ solution can both effectively arrest ECC. The results of this study will add to the clinical trial information on the use of $\mathrm{AgNO}_{3}$ and $\mathrm{NaF}$ and will help to determine whether $25 \% \mathrm{AgNO}_{3}$ solution and $5 \% \mathrm{NaF}$ varnish are as effective as $38 \%$ SDF solution in arresting childhood caries. Lower concentrations of silver and fluoride are contained in $25 \% \mathrm{AgNO}_{3}$ solution and $5 \% \mathrm{NaF}$ varnish, respectively, than in $38 \%$ SDF solution; therefore, $25 \%$ $\mathrm{AgNO}_{3}$ solution and $5 \% \mathrm{NaF}$ varnish are more favourable for use in young children. Because its use for caries management is painless, simple, low-cost, and approved in many countries, $25 \% \mathrm{AgNO}_{3} / 5 \% \mathrm{NaF}$ could be widely recommended and promoted as an alternative treatment to conventional invasive caries management, particularly among child patients who are too young for conventional dental care.

\section{Trial status}

This randomised clinical trial has been registered in ClinicalTrials.gov (U.S.) under the registration number NCT02019160 on 11 December 2013. The recruitment of participating kindergarten children and their parents is in progress from 15 September 2014.

\section{Additional file}

Additional file 1: Questionnaire survey. (DOCX $15 \mathrm{~kb}$ )

\section{Abbreviations}

$\mathrm{AgNO}_{3}$ : silver nitrate; CPI: Community Periodontal Index; dmfs: decayed, missing, filled surfaces score; ECC: early childhood caries; FDI: World Dental Federation; HKU/HAHKW IRB: The Institutional Review Board of the University of Hong Kong/Hospital Authority Hong Kong West Cluster; NaF: sodium fluoride; SDF: silver diamine fluoride; US: United States; VPI: visible plaque index.

\section{Competing interests}

The authors declare that they have no competing interests.

\section{Authors' contributions}

CHC conceived of the study, participated in its design and coordination, and participated in drafting and revising the manuscript. ECML participated in the coordination of the study, acquisition of data, and in drafting and revising the manuscript. SSG contributed to the study conception, design, and manuscript revision. SKYL and MCMW contributed to the data analysis plan, power calculations, and manuscript revision. All authors critically reviewed the manuscript for intellectual content, approved the final version, and have agreed to be accountable for all aspects of the work.

\section{Acknowledgments}

This research is funded by the General Research Fund (number: 17107315) of the University Grant Council, Hong Kong. 
Received: 2 July 2015 Accepted: 15 September 2015

Published online: 25 September 2015

\section{References}

1. American Academy of Pediatric Dentistry. Definition of early childhood caries. Adopted in 2003, last revised in 2008. http://www.aapd.org/assets/1/ 7/D_ECC.pdf.

2. Dye BA, Tan S, Smith $V$, Lewis B, Barker L, Thornton-Evans G, et al. Trends in oral health status: United States, 1988-1994 and 1999-2004. Vital Health Stat. 2007;248:1-92. Series 11, Data from the national health survey.

3. Chu C. Treatment of early childhood caries: a review and case report. Gen Dent. 1999;48(2):142-8

4. Chu C, Fung D, Lo E. Dental public health: dental caries status of preschool children in Hong Kong. Br Dent J. 1999;187(11):616-20.

5. Chu C-H, Ho P-L, Lo EC. Oral health status and behaviours of preschool children in Hong Kong. BMC Public Health. 2012;12(1):767.

6. Beaglehole R, Editions M. The oral health atlas: mapping a neglected global health issue. FDI World Dental Federation. Switzerland; 2009

7. Chu C, Lo E. Dental caries prevention and treatment for preschool children in China. Chin J Dent Res. 2007;10 Suppl:54-60.

8. Frencken JE, Pilot T, Songpaisan Y, Phantumvanit P. Atraumatic restorative treatment (ART): rationale, technique, and development. J Public Health Dent. 1996;56(3):135-40.

9. Chu C, Mei ML, Lo E. Use of fluorides in dental caries management. Gen Dent. 2009;58(1):37-43. quiz 44-35, 79-80.

10. Braga M, Mendes F, De Benedetto M, Imparato J. Effect of silver diamine fluoride on incipient caries lesions in erupting permanent first molars: a pilot study. J Dent Child. 2009;76(1):28-33.

11. Chu C, Lo E, Lin H. Effectiveness of silver diamine fluoride and sodium fluoride varnish in arresting dentin caries in Chinese pre-school children. J Dent Res. 2002;81(11):767-70.

12. Liu BY, Lo ECM, Chu CH, Lin HC. Randomized trial on fluorides and sealants for fissure caries prevention. J Dent Res. 2012;91:753.

13. Llodra J, Rodriguez A, Ferrer B, Menardia V, Ramos T, Morato M. Efficacy of silver diamine fluoride for caries reduction in primary teeth and first permanent molars of schoolchildren: 36-month clinical trial. J Dent Res. 2005;84(8):721-4.

14. Lo E, Chu C, Lin H. A community-based caries control program for preschool children using topical fluorides: 18-month results. J Dent Res. 2001;80(12):2071-4.

15. Yee R, Holmgren C, Mulder J, Lama D, Walker D, van Palenstein HW. Efficacy of silver diamine fluoride for arresting caries treatment. J Dent Res. 2009;88(7):644-7.

16. Chu C, Lo E. Promoting caries arrest in children with silver diamine fluoride: a review. Oral Health Prev Dent. 2008;6(4):315-21.

17. Rosenblatt A, Stamford T, Niederman R. Silver diamine fluoride: a caries 'ilver-fluoride bullet'. J Dent Res. 2009;88(2):116-25.

18. Milgrom $\mathrm{P}, \mathrm{Chi}$ DL. Prevention-centered caries management strategies during critical periods in early childhood. J Calif Dent Assoc. 2011;39(10):735-41.

19. dos Santos VE, de Vasconcelos F, Ribeiro AG, Rosenblatt A. Paradigm shift in the effective treatment of caries in schoolchildren at risk. Int Dent J. 2012;62(1):47-51

20. Gotjamanos T, Afonso F. Unacceptably high levels of fluoride in commercial preparations of silver fluoride. Aust Dent J. 1997;42(1):52-3.

21. Taylor BJ. Switzerland diamine fluoride. Membership Matters. 2013;19:3.

22. Chu C, Lo E. A review of sodium fluoride varnish. Gen Dent. 2005;54(4):247-53.

23. Duffin S. Back to the future: the medical management of caries introduction. J Calif Dent Assoc. 2012;40(11):852-8.

24. Mei ML, Zhao IS, Chu CH. Action of silver nitrate and sodium fluoride on dentine demineralisation. [Abstract] J Dent Res. 2015. 94 (Spec Iss A):2910, p203.

25. Zhao IS, Mei ML, Chu CH. Preventing dentine collagen degradation by silver nitrate and sodium fluoride. [Abstract] J Dent Res. 2015. 94 (Spec Iss A):S2911, p203.

26. Piaggio G, Elbourne DR, Pocock SJ, Evans SJ, Altman DG, Group C. Reporting of noninferiority and equivalence randomized trials: extension of the CONSORT 2010 statement. JAMA. 2012;308(24):2594-604.

27. Zhi QH, Lo ECM, Lin HC. Randomized clinical trial on effectiveness of silver diamine fluoride and glass ionomer in arresting dentine caries in preschool children. J Dent. 2012;40(11):962-7.

28. Lo EC-M, Loo EK-Y, Lee C. Dental health status of Hong Kong preschool children. Hong Kong Dent J. 2009;6(1):6-12.

\section{Submit your next manuscript to BioMed Central and take full advantage of:}

- Convenient online submission

- Thorough peer review

- No space constraints or color figure charges

- Immediate publication on acceptance

- Inclusion in PubMed, CAS, Scopus and Google Scholar

- Research which is freely available for redistribution

Submit your manuscript at www.biomedcentral.com/submit 